\title{
HOMOTOPY RIGIDITY OF LINEAR ACTIONS: CHARACTERS TELL ALL
}

\section{BY ARUNAS LIULEVICIUS ${ }^{1}$}

Our aim is to present a striking rigidity phenomenom in unitary representations of compact groups. Let $U=U(n)$ be a unitary group and $H$ a closed subgroup of $U$. The homogeneous space $U / H$ is a smooth manifold with a smooth action $\lambda$ of $U$ induced by left multiplication. If $\alpha: G \rightarrow U$ is a representation of the compact group $G$, then $\lambda \circ(\alpha \times 1): G \times U / H \rightarrow U / H$ is an action of $G$ on $U / H$, and we denote this $G$-structure by $(U / H, \alpha)$. Such actions of $G$ on $U / H$ are called linear actions. We shall give a complete description of the $G$-homotopy types of linear actions on $U / H$ for a certain class of $\boldsymbol{H}$. To motivate our results we shall first examine some obvious $G$-equivalences of linear actions.

If $X$ is a $U$-space, then the set of $U$-maps $\operatorname{Map}_{U}(U / H, X)$ is in one-to-one correspondence with elements $x \in X$ such that $U_{x} \supset H$, where $U_{x}=\{u \in$ $U \mid u x=x\}$ is the isotropy group of the action at $x$. For example, if $a \in U$ then the element $a H$ in $U / H$ has isotropy group $a H^{-1}$ and the $U$-map $f$ : $U / a H a^{-1} \rightarrow U / H$ given by $f\left(u a H a^{-1}\right)=u a H$ is a $U$-equivalence. Indeed if $H$ and $K$ are closed subgroups of $U$ then $U / H$ and $U / K$ are $U$-equivalent if and only if $K=a \mathrm{Ha}^{-1}$ for a suitable $a \in U$. Suppose $\alpha, \gamma: G \rightarrow U$ are representations such that there exists an $a \in U$ such that $\gamma(g)=a \alpha(g) a^{-1}$ for all $g \in G$ (we say that $\gamma$ is similar to $\alpha$ ). The map $k:(U / H, \alpha) \rightarrow$ $(U / H, \gamma)$ given by $k(u H)=a u H$ is a $G$-equivalence. Indeed, $k$ is the composition of the $G$-equivalence $(U / H, \alpha) \rightarrow\left(U / a \mathrm{Ha}^{-1}, \gamma\right)$ induced by conjugation with $a$ in $U$ and the $U$-equivalence (hence $G$-equivalence!) $f$ : $\left(U / a \mathrm{Ha}^{-1}, \gamma\right) \rightarrow(U / H, \gamma)$. Thus similarity of representations gives us $G$ equivalences of the associated linear actions on $U / H$. Here is another obvious way of obtaining $G$-equivalences: let $c: U \rightarrow U$ be conjugation by unitary matrices $c(a)=\bar{a}$; then if $c(H)=H$, we obtain a $G$-equivalence $c$ : $(U / H, \alpha) \rightarrow(U / H, \bar{\alpha})$ where $\bar{\alpha}=c \circ \alpha$ is the representation conjugate to $\alpha$.

It is too much to hope that $(U / H, \alpha)$ is $G$-homotopy equivalent to $(U / H, \beta)$ if and only if $\beta$ or $\bar{\beta}$ is similar to $\alpha$. For example, if $H$ is a subgroup of maximal rank in $U$ and $C$ is the center of $U$ then $C \subset H$ and $C$ acts trivially on $U / H$, so if we let $P(U)=U / C$ be the projective unitary group (with $q: U \rightarrow P(U)$ the quotient map), then the standard left action $\lambda$ of $U$ on $U / H$ induces an action of $P(U)$ on $U / H$, and it is the similarity class of the projective representation $q \circ \alpha: G \rightarrow P(U)$ which matters. We have: if $\alpha, \beta: G \rightarrow U$ are representations and $\chi: G \rightarrow S^{1}=C$ is a homomorphism such that $\beta$ or $\bar{\beta}$ is similar to $\chi \alpha$ then $(U / H, \alpha)$ is $G$-equiva-

An invited address presented at the 745th meeting of the American Mathematical Society, Evanston, Illinois, April 16, 1977; received by the editors July 18, 1977.

AMS (MOS) subject classifications (1970). Primary 57E10, 57E25, 55D15; Secondary 55B15, 57D20, $22 \mathrm{C} 05$.

Key words and phrases. Representation, linear action, $G$-homotopy, cohomology, Picard group.

${ }^{1}$ Work supported in part by NSF grant MCS 75-08280. 
lent to $(U / H, \beta)$, and indeed through a map which is induced by an $R$-linear map of $R^{2 n}$ (the underlying real vector space of the complex vector space $C^{n}$ on which $U=U(n)$ acts in the standard way). The reader would expect to find more $G$-equivalences of linear actions if we drop linearity, and yet more $G$-homotopy equivalences. The surprise is that if we make a mild restriction on $H$ then we find that linear actions of $G$ on $U / H$ are rigid under homotopy: $(U / H, \alpha)$ is $G$-homotopy equivalent to $(U / H, \beta)$ if and only if they are $G$-equivalent through an $R$-linear map. Here is a sample result:

THEOREM 1 (HOMOTOPY RIGIDITY OF LINEAR ACTIONS). If $\boldsymbol{H}$ is a subgroup of $U=U(n)$ conjugate to $U(n-k) \times T^{k}$, where $T^{k}$ is the $k$-torus and $n \geqslant 2 k$, $\alpha, \beta: G \rightarrow U$ representations of a compact group $G$, then a $G$-map $f:(U / H, \alpha)$ $\rightarrow(U / H, \beta)$ exists with $f: U / H \rightarrow U / H$ a homotopy equivalence if and only if there is a linear character $\chi: G \rightarrow S^{1}$ and $\beta$ or $\bar{\beta}$ is similar to $\chi \alpha$.

We should point out that the condition $n \geqslant 2 k$ is not necessary: for example, homotopy rigidity of linear actions holds for $U(5) / U(2) \times T^{3}$ and for $U(6) / U(2) \times T^{4}$, but the proof is much more involved. Similarly, the condition that $H$ be conjugate to $U(n-k) \times T^{k}$ is too strong: in [13] we show homotopy rigidity of linear actions on $U(m+n+1) / U(m) \times U(n)$ $\times U(1)$ for $m n \geqslant m+n+1$. The right level of generality for our current approach seems to be the following: let us call a subgroup $H$ of $U$ friendly if $H$ is closed, connected, of maximal rank in $U=U(n)$ and there exists a nonzero vector $v \in C^{n}$ such that $h v=\lambda(h) v$ for some linear character $\lambda$ : $h \rightarrow S^{1}$; indeed we assume $H$ is conjugate to a subgroup $U\left(n_{1}\right) \times \cdots \times$ $U\left(n_{k}\right) \subset U(n)$ with $n_{1} \geqslant \cdots \geqslant n_{k}=1$ and $n_{1}+\cdots+n_{k}=n$ (see Borel and Siebenthal [7]). We shall outline a strategy for proving

CONJECTURe A. If $H$ is a friendly subgroup of $U$ then linear actions of a compact group $G$ on $U / H$ are rigid under homotopy.

Indeed one can conjecture that linear actions of $G$ are rigid for $U / H$ where $H$ is connected of maximal rank. This is work in progress with Wu-Yi Hsiang.

An immediate consequence of our homotopy rigidity result is that the $G$-homotopy type of $(U / H, \alpha)$ can be read off from the character table of $G$ (characters tell all). For example, if $\alpha, \beta: G \rightarrow U$ are representations and $\mid$ Trace $\alpha(g)|\neq|$ Trace $\beta(g) \mid$ for some element $g \in G$, then $(U / H, \alpha)$ and $(U / H, \beta)$ have distinct $G$-homotopy types. An example of such a situation is given by the alternating group on five letters $A_{5}:$ let $\alpha$ and $\beta$ be the distinct irreducible 3-dimensional unitary representations, $g=(12345)$, then

$$
\operatorname{Tr} \alpha(g)=\frac{1+\sqrt{ } 5}{2} \text { and } \operatorname{Tr} \beta(g)=\frac{1-\sqrt{ } 5}{2},
$$

so $(U / H, \alpha)$ and $(U / H, \beta)$ are not $A_{5}$-homotopy equivalent for any friendly subgroup $H$ of $U=U(3)$. Here, of course, there are no nontrivial linear characters and all characters of $A_{5}$ take real values, so two linear actions $(U / H, \gamma),(U / H, \delta)$ of $A_{5}$ on $U / H$ (with $H$ a friendly subgroup of $U$ ) are $A_{5}$-homotopy equivalent if and only if $\gamma$ is similar to $\delta$. The case of $\alpha$ and $\beta$ is especially interesting since there is an outer automorphism $\varphi: A_{5} \rightarrow A_{5}$ with $\varphi^{*} \alpha=\beta$. Even the cyclic group of order two $G=Z / 2 Z$ gives entertaining examples: if we let 1 denote the trivial representation of $G$ then there exist linear actions $\alpha, \beta, \gamma, \delta$ on $C P^{n}$ such that $\left(C P^{n}, \alpha\right) \approx\left(C P^{n}, \beta\right)$ but $\left(C P^{n}, \alpha\right.$ 
$+1) \approx\left(C P^{n}, \beta+1\right)$, and $\left(C P^{n}, \gamma+1\right) \approx\left(C P^{n}, \delta+1\right)$ but $\left(C P^{n}, \gamma\right) \approx$ $\left(C P^{n}, \delta\right)$, where we have used $\approx$ to indicate $Z / 2 Z$-homotopy equivalence.

In Theorem $1, f$ is not assumed to be a $G$-homotopy equivalence, that is, although there is a homotopy inverse $f^{\prime}: U / H \rightarrow U / H$, we are not saying that such an $f^{\prime}$ can be found which is a $G$-map $f^{\prime}:(U / H, \beta) \rightarrow(U / H, \alpha)$. Indeed, Petrie [15] exhibits a $G$-space $Y$, a linear action $\gamma$ on $U / H=C P^{k}$ and a $G$-map $h: Y \rightarrow\left(C P^{k}, \gamma\right)$ which is a homotopy equivalence such that the induced map is equivariant $K$-theory

$$
h^{!}: K_{G}\left(C P^{k}, \gamma\right) \rightarrow K_{G}(Y)
$$

is not an isomorphism-this means that although $h$ is a homotopy equivalence it is not a G-homotopy equivalence. Our approach is based on the fact that this sort of pathology cannot occur if $Y$ is a complex projective space with a linear action (see [11]): given a $G$-map $h:\left(C P^{n}, \alpha\right) \rightarrow\left(C P^{n}, \beta\right)$ such that $h$ : $C P^{n} \rightarrow C P^{n}$ is a homotopy equivalence, there exists an $R$-linear $G$-equivalence $k:\left(C P^{n}, \alpha\right) \rightarrow\left(C P^{n}, \beta\right)$ such that $h^{!}=k^{!}$(so, in particular, $h^{!}$is an isomorphism).

This report is organized as follows: in the second section, we present an exact sequence on Picard groups of $G$-line bundles and popularize some work of Graeme Segal [19] on cohomology of topological groups. In the third section we examine the case $U / H=C P^{n}$ and show how equivariant $K$ theory allows us to prove the homotopy rigidity theorem for this case. We also examine the general case of $H$ a friendly subgroup of $U$ and show how a result on cohomology automorphisms of $U / H$ implies the homotopy rigidity theorem. The fourth section is devoted to proving the result on automorphisms of $H^{*}(U / H, Z)$, where $H$ is as in Theorem 1 .

A few words about the background of the problem. There is an extensive literature about $G$-maps of spheres with linear action: de Rham [16], Atiyah and Tall [5], Lee and Wasserman [10], Meyerhoff and Petrie [14]. The current project is the result of numerous consultations with Ted Petrie. Thanks also go to J. F. Adams, J. Dupont, H. Glover, W.-Y. Hsiang, P. Landrock, I. Madsen, G. Segal, R. Stong and J. Tornehave for their helpful comments.

2. An exact sequence of Picard groups. Let $X$ be a $G$-space, $\operatorname{Pic}_{G}(X)$ the set of isomorphism classes of complex $G$-line bundles over $X$. We give $\operatorname{Pic}_{G}(X)$ the structure of a group by using the tensor product of line bundles as multiplication. If $X$ is a CW complex, then $H^{1}(X ; Z) \cong\left[X, S^{1}\right]$ and

$$
H^{2}(X ; Z) \cong\left[X, C P^{\infty}\right] \cong \operatorname{Pic}_{E}(X),
$$

where $E \subset G$ is the subgroup consisting of the identity element.

THEOREM 2. If $X$ is a nonempty connected $G$-space and $H^{1}(X: Z)=0$ then the following sequence is exact:

$$
\operatorname{Pic}_{G}(*) \stackrel{c^{!}}{\rightarrow} \operatorname{Pic}_{G}(X) \stackrel{i^{!}}{\rightarrow} \operatorname{Pic}_{E}(X),
$$

where $c: X \rightarrow *$ is the collapsing map onto a point, $i: E \subset G$ the inclusion of the identity subgroup.

Proof. We shall use the technique of Segal's cohomology of groups [19]: if $A$ is an abelian $G$-group ( $G$ compact, $A$ has the compactly generated 
topology) then cohomology groups $H_{G}^{i}(A)$ are defined for all $i \geqslant 0$. The group $H_{G}^{1}(A)$ is the quotient of the group of all crossed homomorphisms $\varphi$ : $G \rightarrow A$ (functions which satisfy $\varphi\left(g g^{\prime}\right)=\varphi(g)+g \cdot \varphi\left(g^{\prime}\right)$ for all $g, g^{\prime}$ in $G$ ) modulo principal crossed homomorphisms (those which have the form $\varphi(g)$ $=g \cdot a-a$ for a fixed $a$ in $A$ ). The pleasant thing about Segal's cohomology is that a short exact sequence $0 \rightarrow A^{\prime} \rightarrow A \rightarrow A^{\prime \prime} \rightarrow 0$ (meaning that $A$ is a principal $A^{\prime}$-bundle with $A^{\prime \prime}$ as base) produces a long exact sequence

$$
\ldots \rightarrow H_{G}^{i}(A) \rightarrow H_{G}^{i}\left(A^{\prime \prime}\right) \stackrel{\delta}{\rightarrow} H_{G}^{i+1}\left(A^{\prime}\right) \rightarrow H_{G}^{i+1}(A) \rightarrow \cdots
$$

If $V$ is a vector space over $R$ then $H_{G}^{i}(V)=0$ for all $i>0$. Given our CW space $X$ we first notice that $H_{G}^{1}\left(\operatorname{Map}\left(X, S^{1}\right)\right)$ is precisely the set of isomorphism classes of $G \times S^{1}$-structures on the projection $\pi_{1}: X \times S^{1} \rightarrow X$, that is, $H_{G}^{1}\left(\operatorname{Map}\left(X, S^{1}\right)\right)=\operatorname{Ker} i^{!}$. Since $H^{1}(X: Z)=0$ we obtain an exact sequence

$$
0 \rightarrow \operatorname{Map}(X, Z) \rightarrow \operatorname{Map}(X, R) \rightarrow \operatorname{Map}\left(X, S^{1}\right) \rightarrow 1,
$$

$\operatorname{Map}(X, Z)=Z$ since $X$ is connected, and the collapsing map $c: X \rightarrow *$ induces a map of exact sequences

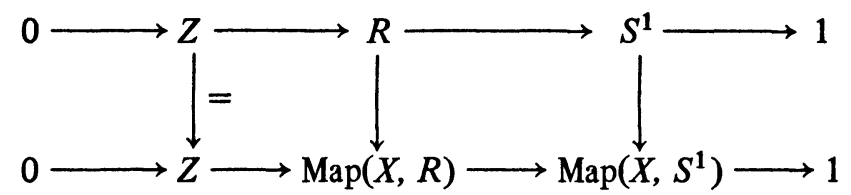

which in turn induces maps of long exact sequences of cohomology groups. We have

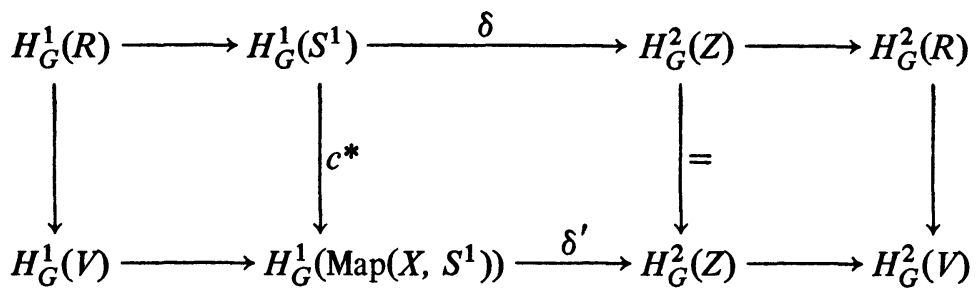

where $V=\operatorname{Map}(X, R)$ is a vector space over $R$, so in both exact sequences the extreme terms are zero, hence $\delta$ and $\delta^{\prime}$ are isomorphisms; thus $c^{*}$ : $H_{G}^{1}\left(S^{1}\right) \rightarrow H_{G}^{1}\left(\operatorname{Map}\left(X, S^{1}\right)\right)=\operatorname{Ker} i^{!}$is an isomorphism, but $H_{G}^{1}\left(S^{1}\right) \cong$ $\operatorname{Pic}_{G}(*) \cong \operatorname{Hom}\left(G, S^{1}\right)$, and under the isomorphism $c^{*}$ corresponds to $c^{!}$, so Theorem 2 is proved.

Notice that $\operatorname{Pic}_{E}(Y) \cong H^{2}(Y ; Z)$ under the isomorphism which assigns to a line bundle $\lambda$ its first Chern class $c_{1}(\lambda)$.

Corollary 3. Let $f: X \rightarrow Y$ be a $G$-map, $X$ connected, $H^{1}(X ; Z)=0$, s a $G$-line bundle over $X, t$ a $G$-line bundle over $Y$. Suppose $f^{*} c_{1}(i t)=c_{1}(i \cdot s)$, then there exists a homomorphism $\chi: G \rightarrow S^{1}$ such that $f^{\prime} t=\chi s$.

Proof. Contemplate $s^{-1} \cdot f^{\prime} t$. We have

$$
c_{1}\left(i^{\prime}\left(s^{-1} \cdot f^{\prime} t\right)\right)=-c_{1}\left(i^{\prime} s\right)+c_{1}\left(i^{\prime} f^{\prime} t\right)=-c_{1}\left(i^{\prime} s\right)+f^{*} c_{1}\left(i^{\prime} t\right)=0,
$$

so $s^{-1} \cdot f^{\prime} t$ is in the kernel of $i^{!}$, hence in the image of $c^{!}$-there exists a linear character $\chi: G \rightarrow S^{1}$ with $c^{\prime} \chi=\chi \cdot 1=s^{-1} \cdot f^{\prime} t$, or $f^{\prime} t=\chi s$, as claimed. 
3. The strategy of proof. Let $C P^{n-1}$ be a complex projective $(n-1)$ dimensional space, $s: S^{2 n-1} \rightarrow C P^{n-1}$ the Hopf bundle over $C P^{n-1}$. If $\gamma$ : $G \rightarrow U=U(n)$ is a representation, $s=s(\gamma):\left(S^{2 n-1}, \gamma\right) \rightarrow\left(C P^{n-1}, \gamma\right)$ defines an element in $\operatorname{Pic}_{G}\left(C P^{n-1}, \gamma\right)$, hence an element in $K_{G}\left(C P^{n-1}, \gamma\right)$ which we still call $s$. Let $R(G)=K_{G}(*)$ be the complex representation ring of $G$, then [3], [18] $K_{G}\left(C P^{n-1}, \gamma\right)$ is a free $R(G)$-module with $1, \ldots, s^{n-1}$ as basis and

$$
s^{n}-\gamma s^{n-1}+\left(\Lambda^{2} \gamma\right) s^{n-2}-\cdots+(-1)^{n} \Lambda^{n} \gamma=0,
$$

where $\Lambda^{i} \gamma$ denotes the $i$ th exterior power of $\gamma$.

Proposition 4. Let $\varphi: K_{G}\left(C P^{n-1}, \beta\right) \rightarrow K_{G}\left(C P^{n-1}, \alpha\right)$ be a homomorphism of $R(G)$-algebras with $\varphi(s(\beta))=\chi s(\alpha)$ for some linear character $\chi: G \rightarrow S^{1}$. Then $\beta$ is similar to $\chi \alpha$.

Proof. Let $s(\alpha)=s, s(\beta)=t$. Then $t$ satisfies

$$
t^{n}-\beta t^{n-1}+\cdots+(-1)^{n} \Lambda^{n} \beta=0 .
$$

Hence applying $\varphi$ we have

$$
\chi^{n} s^{n}-\beta \chi^{n-1} s^{n-1}+\cdots+(-1)^{n} \Lambda^{n} \beta=0,
$$

and multiplying with $\chi^{-n}$ we obtain

$$
s^{n}-\beta \chi^{-1} s^{n-1}+\cdots+(-1)^{n} \Lambda^{n}\left(\beta \chi^{-1}\right)=0 .
$$

But

$$
s^{n}-\alpha s^{n-1}+\cdots+(-1)^{n} \Lambda^{n} \alpha=0
$$

and $K_{G}\left(C P^{n-1}, \alpha\right)$ is $R(G)$-free on $1, \ldots, s^{n-1}$. Comparing the coefficients of $s^{n-1}$ we obtain $\beta \chi^{-1}=\alpha$ in $R(G)$ as claimed.

We shall now show how homotopy rigidity of linear actions on $C P^{n-1}$ follows (compare [11], [12]). Let $f:\left(C P^{n-1}, \alpha\right) \rightarrow\left(C P^{n-1}, \beta\right)$ be a $G$-map so that $f^{*}: H^{*}\left(C P^{n-1} ; Z\right) \rightarrow H^{*}\left(C P^{n-1} ; Z\right)$ is an isomorphism. Let $u=c_{1}(s)=$ $c_{1}(i ! s(\alpha))$, the first Chern class of the Hopf bundle $s$. Then $f^{*} u=u$ or $-u$ since $f^{*}$ is an isomorphism and $H^{2}\left(C P^{n-1} ; Z\right)$ is generated by $u$. If $f^{*} u=$ $-u$, we replace $f$ by $c \circ f$ and $\beta$ by $\bar{\beta}$ (where $c: C P^{n-1} \rightarrow C P^{n-1}$ is induced by conjugation in $U=U(n)$ ), so we may assume $f^{*} u=u$, that is $f^{*} c_{1}\left(i^{\prime} t\right)=$ $c_{1}(i ' s)$. We apply Corollary 3: there exists a linear character $\chi: G \rightarrow S^{1}$ such that $f^{\prime} t=\chi s$. Applying Proposition 4 to $\varphi=f^{!}$we obtain that $\beta$ is similar to $\chi \alpha$. Recalling that we may have had to replace our original $\beta$ by $\bar{\beta}$ to obtain $f^{*} u=u$ we obtain the homotopy rigidity result for linear actions on $C P^{n-1}$.

We build our approach to linear actions on $U / H$ on this special case of $C P^{n-1}$. Suppose $H$ is a friendly subgroup of $U=U(n)$; there exists a nonzero vector $v \in C^{n}$ such that $h v=\lambda(h) v$ for all $h \in H$ for some linear character $\lambda$. We define a map $\pi: U / H \rightarrow C P^{n-1}$ by $\pi(u H)=[u v]$. If $\alpha$ : $G \rightarrow U$ is a representation then $\pi$ is a $G$-map $\pi_{\alpha}:(U / H, \alpha) \rightarrow\left(C P^{n-1}, \alpha\right)$.

PRoposition 5. If $H$ is a friendly subgroup of $U=U(n)$ and $\pi_{\alpha}$ is as above, then $\pi_{\alpha}^{!}: K_{G}\left(C P^{n-1}, \alpha\right) \rightarrow K_{G}(U / H, \alpha)$ is a monomorphism. 
Proof. We may as well assume $H=U\left(n_{1}\right) \times U\left(n_{2}\right) \times \cdots \times U\left(n_{k}\right)$ with $n_{k}=1$ and $v=\varepsilon_{n}$, the last vector in the standard basis of $C^{n}$, then $\pi$ is induced by the inclusion $H \subset U(n-1) \times U(1)$. Let $T=U(1) \times \cdots \times$ $U(1)$ be the standard $n$-torus of $U$ consisting of diagonal matrices, then $T \subset H \subset U$ induces a commutative diagram of projections

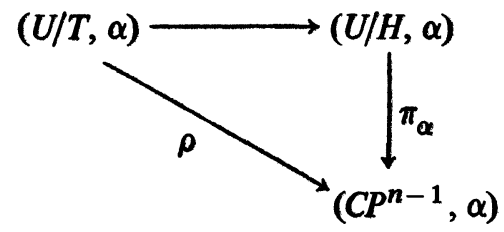

and since $\rho !$ is a monomorphism (see [18]), so is $\pi_{\alpha}^{!}$.

Now let $\alpha, \beta: G \rightarrow U$ be representations, $s=s(\alpha), t=s(\beta)$ the $G$-Hopf bundles on $\left(C P^{n-1}, \alpha\right)$ and $\left(C P^{n-1}, \beta\right)$, respectively. Let $f:(U / H, \alpha) \rightarrow$ $(U / H, \beta)$ be a $G$-map such that $f: U / H \rightarrow U / H$ is a homotopy equivalence. Let $u=c_{1}\left(i^{\prime} \pi_{\alpha}^{\prime} s\right)=c_{1}\left(i^{\prime} \pi_{\beta}^{!} t\right)$. If $f^{*} u=u$, then as before Corollary 3 says that there exists a linear character $\chi: G \rightarrow S^{1}$ such that $f^{\prime} \pi_{\beta}^{!} t=\chi \pi_{\alpha}^{!} s=\pi_{\alpha}^{!}(\chi s)$. Thus $f^{!}$maps the image of $\pi_{\beta}^{!}$into the image of $\pi_{\alpha}^{!}$. Since $\pi_{\alpha}^{!}$is a monomorphism, we may define

$$
\varphi=\left(\pi_{\alpha}^{!}\right)^{-1} f^{!} \pi_{\beta}^{!}: K_{G}\left(C P^{n-1}, \beta\right) \rightarrow K_{G}\left(C P^{n-1}, \alpha\right)
$$

which, of course, is a map of $R(G)$-algebras and $\varphi(t)=\chi s$, so Proposition 4 says that $\beta$ is similar to $\chi \alpha$. The catch, of course, is that there is no reason to expect that $f^{*} u$ is equal to $u$, so we have to do more work.

The group of $U$-maps $\operatorname{Map}_{U}(U / H, U / H)$ is isomorphic to $N_{U}(H) / H$, where $N_{U}(H)=\left\{a \in U \mid a H a^{-1}=H\right\}$ is the normalizer of $H$ in $U$ (see Bredon [8], Samelson [17]). If $\gamma: G \rightarrow U$ is a representation and $k: U / H \rightarrow$ $U / H$ is a $U$-map, then $k:(U / H, \gamma) \rightarrow(U / H, \gamma)$ is a $G$-map. Let $c: U \rightarrow U$ be given by $c(u)=\bar{u}$, the matrix with complex conjugate entries. We have chosen $H$ in its conjugacy class so that $c(H)=H$, hence $c:(U / H, \gamma) \rightarrow$ $(U / H, \bar{\gamma})$ is a $G$-map. We have a homomorphism

$$
\psi: N_{U}(H) / H \times Z / 2 Z \rightarrow \operatorname{Aut}\left(H^{*}(U / H ; Z)\right)
$$

given by $\psi(k, t)=k^{*} \circ c^{t *}$. Stated in another way: the homomorphism $\psi$ defines an action of $N_{U}(H) / H \times Z / 2 Z$ on $H^{*}(U / H ; Z)$. Of course the group Homeq $(U / H)$ of all homotopy classes of homotopy equivalences of $U / H$ also acts on $H^{*}(U / H ; Z)$ by taking induced homomorphisms in cohomology. We now state several related conjectures.

CONJECTURE B. Let $H$ be a friendly subgroup of $U=U(n), \pi: U / H \rightarrow$ $C P^{n-1}$ the standard map, $u=\pi^{*} c_{1}(s)$, where $s$ is the Hopf bundle on $C P^{n-1}$, then the orbit of $u$ under $N_{U}(H) / H \times Z / 2 Z$ is the same as the orbit of $u$ under Homeq $(U / H)$.

Proposition 6. Conjecture B implies Theorem 1 (homotopy rigidity of linear actions on $U / H)$.

Proof. We keep the notation of our earlier discussion: let $\alpha, \beta: G \rightarrow U=$ $U(n)$ be representations, $f:(U / H, \alpha) \rightarrow(U / H, \beta)$ a $G$-map such that $f$ : 
$U / H \rightarrow U / H$ is a homotopy equivalence, $u=\pi^{*} c_{1}(s)$. According to Conjecture B there exists an element $k$ of $N_{U}(H) / H \times Z / 2 Z$ such that $k^{*} f^{*} u=u$. Replace $f$ by $f \circ k$ (here we may have to replace $\alpha$ by $\bar{\alpha}$ if conjugation is involved). Then $f^{*} u=u$, so $i f^{\prime} \pi_{\beta}^{!} t=i^{!} \pi_{\alpha}^{!} s$, and since $U / H$ is connected and simply connected, we obtain from Corollary 3 a linear character $\chi: G \rightarrow S^{1}$ such that $f^{\prime} \pi_{\beta}^{!} t=\chi \pi_{\alpha}^{!}(s)$. So now letting $\varphi=\pi_{\alpha}^{!-1} f^{\prime} \pi_{\beta}^{!}$ we can apply Proposition 4 to conclude that $\beta$ is similar to $\chi \alpha$.

We shall prove an even stronger result for a multitude of subgroups of $U$ :

Conjecture C. The map $\psi$ is an isomorphsm of $N_{U}(H) / H \times Z / 2 Z$ onto the group of all algebra isomorphisms of $H^{*}(U / H ; Z)$ if $H$ is a friendly subgroup of $U=U(n)$ and $n \geqslant 3$.

Notice that $U(2) / T^{2} \approx S^{2}$, and in this case $\psi$ has a cyclic group or order 2 as a kernel. If $n \geqslant 3, \psi$ is a monomorphism.

Let us boldly walk even further on the limb: the following algebraic conjecture implies Conjecture $\mathrm{B}$ (and Conjecture $\mathrm{C}$ in a lot of cases).

CONJECTURE D. Let $T$ be the standard torus of $U=U(n),\left\{\varepsilon_{1}, \varepsilon_{2}, \ldots, \varepsilon_{n}\right\}$ the standard basis for $C^{n}$, let $\pi_{i}: U / T \rightarrow C P^{n-1}$ for $i=1, \ldots, n$ be given by $\pi_{i}(u T)=\left[u \varepsilon_{i}\right], s$ the Hopf bundle on $C P^{n-1}$, let $x_{i}=\pi_{i}^{*} c_{1}(s)$. If $x \in$ $H^{2}(U / T ; Z)$ and $x^{n}=0$ then there exists an integer $a$ and an $i$ in $\{1,2, \ldots, n\}$ such that $x=a x_{i}$.

The algebraic data are easy to state: $H^{*}(U / T ; Z)=Z\left[x_{1}, \ldots, x_{n-1}\right]$ modulo the ideal $I_{n}=\left(h_{2}, \ldots, h_{n}\right)$, where $h_{i}$ is the sum of all monomials of degree $i$ in $x_{1}, \ldots, x_{n-1}$ (see Borel [6])-for example, for $n=4, h_{2}=x_{1}^{2}+$ $x_{1} x_{2}+x_{1} x_{3}+x_{2}^{2}+x_{2} x_{3}+x_{3}^{2}$. It is important to notice that $n-1$ appears above, not $n$-indeed $x_{n}=-x_{1}-x_{2}-\cdots-x_{n-1}$. The group $N_{U}(T) / T$ is $S_{n}$, the symmetric group on $n$ letters which acts on $H^{*}(U / T ; Z)$ by permuting the $x_{1}, \ldots, x_{n}$. Conjecture $\mathrm{D}$ is trivial to prove for $n=3$. For $n=4$, the algebra is already delightfully complicated and a hint is helpful: examine the solutions of $x^{4}=0$ first over $Z / 3 Z$ and then exploit the fact that multiplication by $x_{1}$ from $H^{6}\left(U(4) / T^{4} ; Z / 3 Z\right)$ to $H^{8}\left(U(4) / T^{4} ; Z / 3 Z\right)$ has kernel of dimension one to show that if $x_{1}+y$ is a solution of $x^{4}=0$ over $Z$ and $y=b x_{2}+c x_{3}$ then for all natural numbers $k$ we have $3^{k} \mid y$ implies $3^{k+1} \mid y$, so $y=0$.

The limb is beginning to creak ominously, but let's take one more step:

CONJECTURE E. If $H$ is a connected subgroup of maximal rank of $U=$ $U(n)$ and Homeq $(U / H)$ is the group of homotopy classes of homotopy equivalences of $U / H$ then $N_{U}(H) / H \times Z / 2 Z$ is a normal subgroup of Homeq $(U / H)$ if $n \geqslant 3$.

One reason for thinking wishfully about Conjecture $\mathrm{E}$ is that it would give a beautifully simple proof of Conjecture $\mathrm{C}$ for $H=T$, the maximal torus of $U(n)$ and Homeq $(U / H)$ is the group of homotopy classes of homotopy equivalences of $U / H$ then $N_{U}(H) / H \times Z / 2 Z$ is a normal subgroup of Homeq $(U / H)$ if $n \geqslant 3$.

4. Algebra automorphisms of $H^{*}(U / H ; Z)$. We shall prove Conjecture $\mathrm{C}$ for $U=U(n), H=U(n-k) \times T^{k}, n \geqslant \max \{2 k, k+2\}$. As before, let $\left\{\varepsilon_{1}, \varepsilon_{2}, \ldots, \varepsilon_{n}\right\}$ be the standard basis of $C^{n}$ and let $\pi_{i}: U / H \rightarrow C P^{n-1}$ be the projection $\pi_{i}(u H)=\left[u \varepsilon_{n-k+i}\right]$ for $i=1, \ldots, k$. Let $y \in H^{2}\left(C P^{n-1} ; Z\right)$ be the Chern class of the Hopf bundle and let $x_{i}=\pi_{i}^{*}(y)$; then $H^{*}(U / H$; 
$Z)=Z\left[x_{1}, \ldots, x_{k}\right] / I$, where the ideal $I=\left(h_{n-k+1}, \ldots, h_{n}\right)$ and $h_{j}$ is the sum of all monomials of degree $j$ in $x_{1}, \ldots, x_{k}$. A free basis for $H^{*}$ is given by $x^{E}=x_{1}^{e_{1}} x_{2}^{e_{2}} \ldots x_{k}^{e_{k}}$, where $0 \leqslant e_{i}<n-k+i$ (see Borel [6]). The group $N_{U}(H) / H$ is $S_{k}$, the symmetric group on $k$ letters, and it acts on $H^{*}(U / H$; $Z$ ) by permuting $x_{1}, \ldots, x_{k}$. We examine the case of $k=2$ more closely.

LEMMA 7. If $u=a x_{1}+b x_{2}$ is an element in $H^{2}\left(U(m+2) / U(m) \times T^{2}\right.$; $Z$ ) with $u^{2 m}=0$, then either $a=0$ or $b=0$.

Proof. We first claim that if both $a$ and $b$ are nonzero and $u^{2 m+1}=0$ then $a=b$. Notice that $x_{1}^{m+2}=x_{2}^{m+2}=0$ (since both come from $C P^{m+1}$ ) and $H^{4 m+2}$ has $x_{1}^{m} x_{2}^{m+1}$ as basis. Moreover, $x_{1}^{m+1} x_{2}^{m}=-x_{1}^{m} x_{2}^{m+1}$. We have

$$
\begin{aligned}
0 & =u^{2 m+1}=\left(a x_{1}+b x_{2}\right)^{2 m+1} \\
& =\left(\begin{array}{c}
2 m+1 \\
m+1
\end{array}\right) a^{m+1} b^{m} x_{1}^{m+1} x_{2}^{m}+\left(\begin{array}{c}
2 m+1 \\
m
\end{array}\right) a^{m} b^{m+1} x_{1}^{m} x_{2}^{m+1},
\end{aligned}
$$

so $a \neq 0, b \neq 0$ implies $a=b$. If now, in addition, $u^{2 m}=0$, then we have

$$
\begin{aligned}
0= & \left(\begin{array}{c}
2 m \\
m+1
\end{array}\right) a^{2 m} x_{1}^{m+1} x_{2}^{m-1}+\left(\begin{array}{c}
2 m \\
m
\end{array}\right) a^{2 m} x_{1}^{m} x_{2}^{m} \\
& +\left(\begin{array}{c}
2 m \\
m-1
\end{array}\right) a^{2 m} x_{1}^{m-1} x_{2}^{m+1},
\end{aligned}
$$

but $H^{4 m}$ has $\left\{x_{1}^{m} x_{2}^{m}, x_{1}^{m-1} x_{2}^{m+1}\right\}$ as basis and

$$
x_{1}^{m+1} x_{2}^{m-1}=-x_{1}^{m} x_{2}^{m}-x_{1}^{m-1} x_{2}^{m+1},
$$

so the above sum reduces to

$$
0=\left\{\left(\begin{array}{c}
2 m \\
m
\end{array}\right)-\left(\begin{array}{c}
2 m \\
m+1
\end{array}\right)\right\} a^{2 m} x_{1}^{m} x_{2}^{m}
$$

so since $\left(\begin{array}{c}2 m \\ m\end{array}\right) \neq\left(\begin{array}{c}2 m \\ m+1\end{array}\right)$ for all $m$ it follows that $a=0$, a contradiction to our temporary hypothesis that $a \neq 0$ and $b \neq 0$.

CoROLlary 8. Let $v \in H^{2}\left(U(m+k) / U(m) \times T^{k} ; Z\right)$ be an element such that $v^{m+k}=0$. If $m \geqslant k$ then $v=a x_{i}$ for some $i$ in $\{1, \ldots, k\}$.

Proof. By applying a suitable element of $S_{k}$ we can assume that the coefficient of $x_{1}$ is nonzero. We wish to show that the coefficient of $x_{i}$ for $i \neq 1$ is zero-and, of course, it is sufficient to prove this for $i=2$. Consider the standard map

$$
j: U(m+2) / U(m) \times T^{2} \rightarrow U(m+k) / U(m) \times T^{k}
$$

induced by the standard inclusion $C^{m+2} \subset C^{m+k}$ under which $j^{*} x_{1}=x_{1}$, $j^{*} x_{2}=x_{2}, j^{*} x_{i}=0$ for $i>2$. Inspect $u=j^{*} v$; then $u^{m+k}=0, m+k \leqslant 2 m$; hence $a \neq 0$ implies that the coefficient of $x_{2}$ is zero.

We are now ready to prove Conjecture $\mathrm{C}$ for $U(m+k) / U(m) \times T^{k}$.

THEOREM 9. If $n \geqslant \max \{2 k, k+2\}, U=U(n), H=U(n-k) \times T$, then the map $\psi$ is an isomorphism of $N_{U}(H) / H \times Z / 2 Z$ onto the group of all algebra isomorphisms of $H^{*}(U / H ; Z)$.

Proof. We first prove that $\psi$ is onto. Let $\varphi: H^{*}(U / H ; Z) \rightarrow H^{*}(U / H ; Z)$ 
be an algebra automorphism; then $\varphi\left(x_{1}\right)=u$ is an element with $u^{n}=0$ (this since $x_{i}^{n}=0$ ) and because $n \geqslant 2 k$, Corollary 8 is applicable, so $u=a x_{i}$ for some $i$ and $a=1$ or -1 . By using elements of $N_{U}(H) / H=S_{k}$ we can normalize $\varphi$ (using $c^{*}$ if necessary) to have $\varphi\left(x_{1}\right)=x_{1}$. We claim $\varphi$ is the identity. If not, use $S_{k}$ to arrange $\varphi\left(x_{2}\right)=-x_{2}$. Now consider $\varphi$ as an automorphism of $Z\left[x_{1}, \ldots, x_{k}\right]$ (remember: there are no relations among the generators in $H^{2}$ ). The relations in grading $2 n-2 k+2$ are generated by $h_{n-k+1}$ so we must have $\varphi h_{n-k+1}= \pm h_{n-k+1}$, but $\varphi\left(x_{1}^{n-k+1}\right)=x_{1}^{n-k+1}$ and $\varphi\left(x_{1}^{n-k} x_{2}\right)=-x_{1}^{n-k} x_{2}$, so $\varphi\left(x_{2}\right)=-x_{2}$ is impossible, and we have shown that $\psi$ is onto.

To prove that $\psi$ is one-to-one is even easier: since $m \geqslant 2$ each $\sigma \in$ $N_{U}(H) / H=S_{k}$ maps $x_{1}$ into some $x_{i}, c^{*} x_{1}=-x_{1}$, so the kernel of $\psi$ is contained in $S_{k} \times 0$, but we have already noticed that $\psi \mid S_{k} \times 0$ is faithful, so $\psi$ is one-to-one, as claimed.

\section{BIBLIOGRAPHY}

1. J. F. Adams, Lectures on Lie groups, Benjamin, New York, 1969.

2. M. F. Atiyah, Characters and cohomology of finite groups, Inst. Hautes Etudes Sci. Publ. Math. No. 9 (1961), 23-64.

3. M. F. Atiyah and G. B. Segal, Lectures on equivariant $K$-theory, Mimeographed Notes, Oxford, 1965.

4. _ Equivariant $K$-theory and completion, J. Differential Geometry 3 (1969), 1-18.

5. M. F. Atiyah and D. O. Tall, Group representations, $\lambda$-rings and the J-homomorphism, Topology 8 (1969), 253-297.

6. A. Borel, Sur la cohomologie des espaces fibrés principaux et des espaces homogenes de groupes de Lie compacts, Ann. of Math. (2) 57 (1953), 115-207.

7. A. Borel and J. de Siebenthal, Les sous-groupes fermés de rang maximum des groups de Lie clos, Comment. Math. Helv. 23 (1949), 200-221.

8. G. Bredon, Introduction to compact transformation groups, Academic Press, London and New York, 1972.

9. H. Glover and W. Homer, Endomorphisms of the cohomology ring of a finite Grassmann manifold, Proc. Homotopy Theory Conf. (Northwestern Univ., March 1977), Springer-Verlag, Lecture Notes in Math. (to appear).

10. C. N. Lee and A. Wasserman, On the groups $J O(G)$, Mem. Amer. Math. Soc., no. 159 (1975).

11. A. Liulevicius, Homotopy types of linear G-actions on complex projective spaces, Mat. Institut, Aarhus Univ. Preprint Series 1975/76, No. 14.

12. Characters do not lie. Transformation Groups, Cambridge Univ. Press, 1976, pp. 139-146.

13. , Line bundles, cohomology automorphisms, and homotopy rigidity of linear actions,

Proc. Homotopy Theory Conf. (Northwestern Univ., March 1977), Springer-Verlag, Lecture Notes in Math. (to appear).

14. A. Meyerhoff and T. Petrie, Quasi-equivalence of G-modules, Topology 15 (1976), 69-75.

15. T. Petrie, $A$ setting for smooth $S^{1}$-actions with applications to real algebraic actions on $P\left(C^{4 n}\right)$, Topology 13 (1974), 363-374.

16. G. de Rham, Reidemeister's torsion invariant and rotations of $S^{n}$, Differential Analysis (Bombay Colloquium), Oxford Univ. Press, 1964.

17. H. Samelson, Topology of Lie groups, Bull. Amer. Math. Soc. 58 (1952), 2-37.

18. G. B. Segal, Equivariant $K$-theory, Inst. Hautes Études Sci. Publ. Math. No. 34 (1968), 129-151.

19. Cohomology of topological groups, Symposia Mathematica, vol. IV (INDAM, Rome, 1968/69), pp. 377-387.

Department of Mathematics, University of Chicago, Chicago, Illinois 60637 